\title{
Crater topography on Titan: Implications for landscape evolution
}

\author{
C.D. Neish ${ }^{\mathrm{a}, *}$, R.L. Kirk ${ }^{\mathrm{b}}$, R.D. Lorenz ${ }^{\mathrm{c}}$, V.J. Bray $^{\mathrm{d}}$, P. Schenk ${ }^{\mathrm{e}}$, B.W. Stiles ${ }^{\mathrm{f}}$, E. Turtle ${ }^{\mathrm{c}}$, K. Mitchell ${ }^{\mathrm{f}}$, \\ A. Hayes ${ }^{\mathrm{g}}$, Cassini RADAR Team \\ ${ }^{a}$ NASA Goddard Space Flight Center, Greenbelt, MD 20771, USA \\ ${ }^{\mathrm{b}}$ United States Geological Survey, Astrogeology Science Center, Flagstaff, AZ 86001, USA \\ ${ }^{\mathrm{c}}$ The Johns Hopkins University Applied Physics Laboratory, Laurel, MD 20723, USA \\ ${ }^{\mathrm{d}}$ Lunar and Planetary Laboratory, University of Arizona, Tucson, AZ 85721, USA \\ ${ }^{\mathrm{e}}$ Lunar and Planetary Institute, Houston, TX 77058, USA \\ ${ }^{\mathrm{f} J e t}$ Propulsion Laboratory, California Institute of Technology, Pasadena, CA 91109, USA \\ ${ }^{\mathrm{g}}$ University of California Berkeley, Berkeley, CA 94720, USA
}

\section{A R T I C L E I N F O}

\section{Article history:}

Received 29 March 2012

Revised 1 November 2012

Accepted 22 November 2012

Available online 3 December 2012

\section{Keywords:}

Cratering

Titan

Ganymede

\begin{abstract}
A B S T R A C T
We present a comprehensive review of available crater topography measurements for Saturn's moon Titan. In general, the depths of Titan's craters are within the range of depths observed for similarly sized fresh craters on Ganymede, but several hundreds of meters shallower than Ganymede's average depth vs. diameter trend. Depth-to-diameter ratios are between $0.0012 \pm 0.0003$ (for the largest crater studied, Menrva, $D \sim 425 \mathrm{~km}$ ) and $0.017 \pm 0.004$ (for the smallest crater studied, Ksa, $D \sim 39 \mathrm{~km}$ ). When we evaluate the Anderson-Darling goodness-of-fit parameter, we find that there is less than a $10 \%$ probability that Titan's craters have a current depth distribution that is consistent with the depth distribution of fresh craters on Ganymede. There is, however, a much higher probability that the relative depths are uniformly distributed between 0 (fresh) and 1 (completely infilled). This distribution is consistent with an infilling process that is relatively constant with time, such as aeolian deposition. Assuming that Ganymede represents a close 'airless' analogue to Titan, the difference in depths represents the first quantitative measure of the amount of modification that has shaped Titan's surface, the only body in the outer Solar System with extensive surface-atmosphere exchange.
\end{abstract}

(c) 2012 Elsevier Inc. All rights reserved.

\section{Introduction}

Unique among the icy satellites, Titan's surface shows evidence for extensive modification by fluvial and aeolian processes (Tomasko et al., 2005; Lorenz et al., 2006, 2008; Stofan et al., 2007). These processes act to change the topography of its surface over time, through fluvial erosion, mass wasting, burial by dunes and submergence in seas. Quantifying the extent of this modification is difficult, since the original, un-eroded surface topography is generally unknown. However, fresh craters on icy satellites have well-known shapes and morphologies, which have been determined from extensive studies of the airless worlds of the outer Solar System (e.g., Schenk et al., 2004). By comparing the topography of craters on Titan to similarly sized, relatively pristine analogues on airless bodies, we can obtain one of the few direct measures of the amount of modification that has occurred on Titan.

The best analogues for comparison to Titan are Jupiter's moons Ganymede and Callisto. For large craters (formed in the gravity regime), crater size is dependent on gravity, impact velocity, projec-

\footnotetext{
* Corresponding author.

E-mail address: catherine.d.neish@nasa.gov (C.D. Neish).
}

tile size, and target and projectile density (Holsapple and Housen, 2007). Ganymede and Titan have similar gravity $\left(g \sim 1.4 \mathrm{~m} / \mathrm{s}^{2}\right)$, target density $\left(\rho \sim 1 \mathrm{~g} / \mathrm{cm}^{3}\right)$, and likely projectile density $(\rho \sim 1 \mathrm{~g} /$ $\mathrm{cm}^{3}$ ), and the average impact velocity at Ganymede is twice that at Titan $(20 \mathrm{~km} / \mathrm{s}$ vs. $10.5 \mathrm{~km} / \mathrm{s}$, Zahnle et al., 2003). The gravity on Callisto is also similar to that of Titan $\left(g=1.25 \mathrm{~m} / \mathrm{s}^{2}\right)$, and its average impact velocity is somewhat lower than that on Ganymede $(\langle v\rangle \sim 15 \mathrm{~km} / \mathrm{s})$. However, given that the depths of craters on Callisto are almost indistinguishable from those of Ganymede (Schenk, 2002), and the topographic data set that exists for Callisto is somewhat sparser than that for Ganymede, we focus in this paper solely on comparisons to Ganymede.

In addition to target and projectile properties, the subsurface structure of an icy satellite is important to the shape and morphology of craters, since rheological changes at depth influence the depth-to-diameter ratio of large craters $(D>26 \mathrm{~km})$ (Schenk, 2002). This range of diameters coincides with those most likely to be observed on Titan, as models predict that craters with $D>20 \mathrm{~km}$ were formed by projectiles large enough to be only minimally disrupted by Titan's extended, thick atmosphere (Korycansky and Zahnle, 2005). In the case of Ganymede and Titan, it appears that their outermost layers $(\sim 100 \mathrm{~km})$ are both 
dominated by cold water ice. Titan's long-wavelength topography (Nimmo and Bills, 2010) and Cassini Radio Science Subsystem (RSS) gravity measurements (Iess et al., 2010) both suggest that Titan has a floating, isostatically compensated ice shell with a mean thickness of $\sim 100 \mathrm{~km}$ overlying a subsurface ocean. Ganymede is also thought to possess an $\mathrm{H}_{2} \mathrm{O}-\mathrm{NH}_{3}$ ocean at $\sim 100 \mathrm{~km}$ depth (Spohn and Schubert, 2003).

Cassini RADAR has imaged $\sim 50 \%$ of the surface of Titan at resolutions as good as $350 \mathrm{~m}$ (e.g., Elachi et al., 2005; Hayes et al., 2011), and in this data set, more than 60 potential craters have been identified (Wood et al., 2010; Neish and Lorenz, 2012). Topographic information for these craters has thus far been difficult to obtain. There is only a limited amount of topographic data on Titan in the form of altimetry (Zebker et al., 2009) and stereo ( $\sim 2 \%$ areal coverage, Kirk et al., 2012). Additionally, altimetry requires nadir pointing, and thus is incompatible with simultaneous synthetic aperture radar (SAR) imaging of the surface. Fortunately, a technique known as 'SARTopo' (Stiles et al., 2009) has been developed that estimates surface heights by comparing the calibration of overlapping SAR beams. The Cassini RADAR instrument has five different antenna feeds (or beams), and data from overlapping beams are acquired nearly simultaneously. Surface height can be computed by maximizing the correlation between the received power and the antenna gain pattern at each point along track in the overlap region. This technique is capable of estimating surface heights for most of the SAR-imaged surface of Titan with $\sim 10 \mathrm{~km}$ horizontal resolution and a vertical resolution of tens of meters. It has extended the area over which co-located topography and SAR imagery is available on Titan by at least an order of magnitude.

In this work, we present topography data for several craters on Titan using the SARTopo data set. We compare this topography to similarly sized craters on Ganymede, for which topography has been extracted from shadow length measurements and stereoderived digital elevation models (Schenk, 2002; Bray et al., 2008, 2012). Finally, we make inferences regarding the relative amount of landscape degradation that has occurred on Titan due to erosion and infill.

\section{Observations}

We plotted the position of the SARTopo data over SAR images of every known crater on Titan with $D>20 \mathrm{~km}$. There are roughly 30 such craters (Wood et al., 2010; Neish and Lorenz, 2012), with eight interpreted as 'certain' impact structures. Of these, six 'certain' or 'nearly certain' craters (Table 1 ) and two 'probable' craters had corresponding SARTopo coverage (Fig. 1). Of the six 'certain' craters, five had topographic profiles broadly consistent with craters of their size on other icy worlds, including a central peak in the $39 \mathrm{~km}$ diameter crater Ksa (Kirk et al., 2012; see also Figs. 1b and 2), a central uplift in the $100 \mathrm{~km}$ diameter crater Hano (Figs. $1 \mathrm{~g}$ and 3), and a near-flat profile for the $425 \mathrm{~km}$ diameter crater Menrva (Figs. 1i and 3). Only Soi lacked any recognizable topography (Fig. 4), as did the two 'probable' craters (craters \#43 and \#49 from Wood et al. (2010); Fig. 5). Both of the 'probable' craters are located in Titan's expansive sand seas, so these features are either not impact craters or have been completely infilled with aeolian deposits. Since we cannot unambiguously determine whether these features are craters, we exclude them from the remainder of the depth analysis.

The diameter of each crater was determined from the SAR imagery, and the errors reported in Table 1 represent the natural variations of the craters away from circularity. Height measurements were determined from a $10-\mathrm{km}$ long (along-track) by $w$-km wide (across-track) region of SAR pixels, where $w$ is the width of overlap between the two antenna beams that contribute to a SARTopo profile. The value of $w$ varies depending upon the spacecraft altitude and instrument pointing angles, but is generally on the order of several kilometers. All of the pixels in the 10-by- $w$ km region were then used together with knowledge of the viewing geometry and radar antenna gain patterns to estimate the heights. The alongtrack translation between consecutive measurements is significantly smaller than the $10 \mathrm{~km}$ region of interest (typically $200 \mathrm{~m}$ ), however, making it necessary to subsample tracks to a $10 \mathrm{~km}$ separation distance ( $\sim 50$ data points) to ensure independent height measurements. This was possible for all depth measurements reported here. Note that this method of height derivation tends to reduce the calculated crater depths, since crater rims are averaged with adjacent, lower topography and crater floors are averaged with adjacent, higher topography (see Fig. 6). These measurements could therefore be considered lower limits.

For the six 'certain' craters, we then calculated depth, $d=h_{1}-h_{2}$, by taking the difference between the highest point on the crater rim and the lowest point on the crater floor, on both sides of the crater, $d_{1}$ and $d_{2}$ (Fig. 6). Systematic errors in height, $\delta h_{i}$, were propagated throughout the analysis. These errors were determined from radar instrument noise and viewing geometry, and are summarized in Table 1 of Stiles et al. (2009). For the depth measurements, we used the topographic profile closest to the midpoint of the crater. However, in several cases, only profiles near the edge of the crater were available (e.g., Sinlap, Afekan). The depth measurements therefore assume that the depth is constant across the crater floor. If this assumption is incorrect, these values represent lower limits for the depth of the craters.

Table 1

Depth-to-diameter ratio for seven 'certain' or 'nearly certain' craters on Titan.

\begin{tabular}{|c|c|c|c|c|c|c|}
\hline Crater & Diameter, $D(\mathrm{~km})$ & Depth, $d(\mathrm{~m})$ & $d / D$ & Technique & Relative depth, $R^{\mathrm{b}, \mathrm{c}}$ & Relative depth, $R^{\mathrm{e}}$ \\
\hline \multirow[t]{2}{*}{ Ksa } & \multirow[t]{2}{*}{$39 \pm 2$} & $660_{-170}^{+170}$ & $0.017_{-0.004}^{+0.004}$ & SARTopo & $0.24_{-0.20}^{+0.20}$ & $0.42_{-0.15}^{+0.15}$ \\
\hline & & $750 \pm 175$ & $0.019 \pm 0.005$ & Stereo $^{\mathrm{a}}$ & $0.13_{-0.20}^{+0.20}$ & $0.34_{-0.15}^{+0.15}$ \\
\hline Momoy & $40 \pm 1$ & $680 \pm 100$ & $0.017 \pm 0.003$ & Autostereo & $0.22_{-0.11}^{+0.20}$ & $0.40_{-0.09}^{+0.09}$ \\
\hline Soi & $78 \pm 2$ & $110 \pm 100$ & $0.001 \pm 0.001$ & SARTopo & $0.90_{-0.09}^{+0.09}$ & $0.89_{-0.10}^{+0.09}$ \\
\hline \multirow[t]{2}{*}{ Sinlap } & \multirow[t]{2}{*}{$82 \pm 2$} & $640_{-150}^{+160}$ & $0.008_{-0.002}^{+0.002}$ & SARTopo & $0.43_{-0.13}^{+0.09}$ & $0.36_{-0.15}^{+0.16}$ \\
\hline & & $700 \pm 100$ & $0.009 \pm 0.001$ & Autostereo & $0.38_{-0.09}^{+0.09}$ & $0.30_{-0.10}^{+0.10}$ \\
\hline Hano & $100 \pm 5$ & $525_{-95}^{+105}$ & $0.005_{-0.001}^{+0.001}$ & SARTopo & $0.56_{-0.08}^{+0.09}$ & $0.46_{-0.10}^{+0.11}$ \\
\hline Afekan & $115 \pm 5$ & $455_{-180}^{+175}$ & $0.004_{-0.002}^{+0.002}$ & SARTopo & $0.62_{-0.15}^{+0.15 d}$ & $0.52_{-0.19}^{+0.19}$ \\
\hline Menrva & $425 \pm 25$ & $490_{-120}^{+110}$ & $0.0012_{-0.0003}^{+0.0003}$ & SARTopo & $\mathrm{N} / \mathrm{A}^{-0.15}$ & $\mathrm{~N} / \mathrm{A}$ \\
\hline
\end{tabular}

\footnotetext{
a See Kirk et al. (2012). Ganymede.

c Ganymede crater depths from Table 4 in Bray et al. (2012).

d Assumed to have the same depth as a $D=100 \mathrm{~km}$ crater.

e Ganymede crater depths from Fig. 2b in Schenk (2002).
}

b Relative depth is defined as $R(D)=1-d_{t}(D) / d_{g}(D)$, where $d_{t}(D)$ is the depth of a crater with diameter $D$ on Titan, and $d_{g}(D)$ is the depth of a crater with diameter $D$ on 


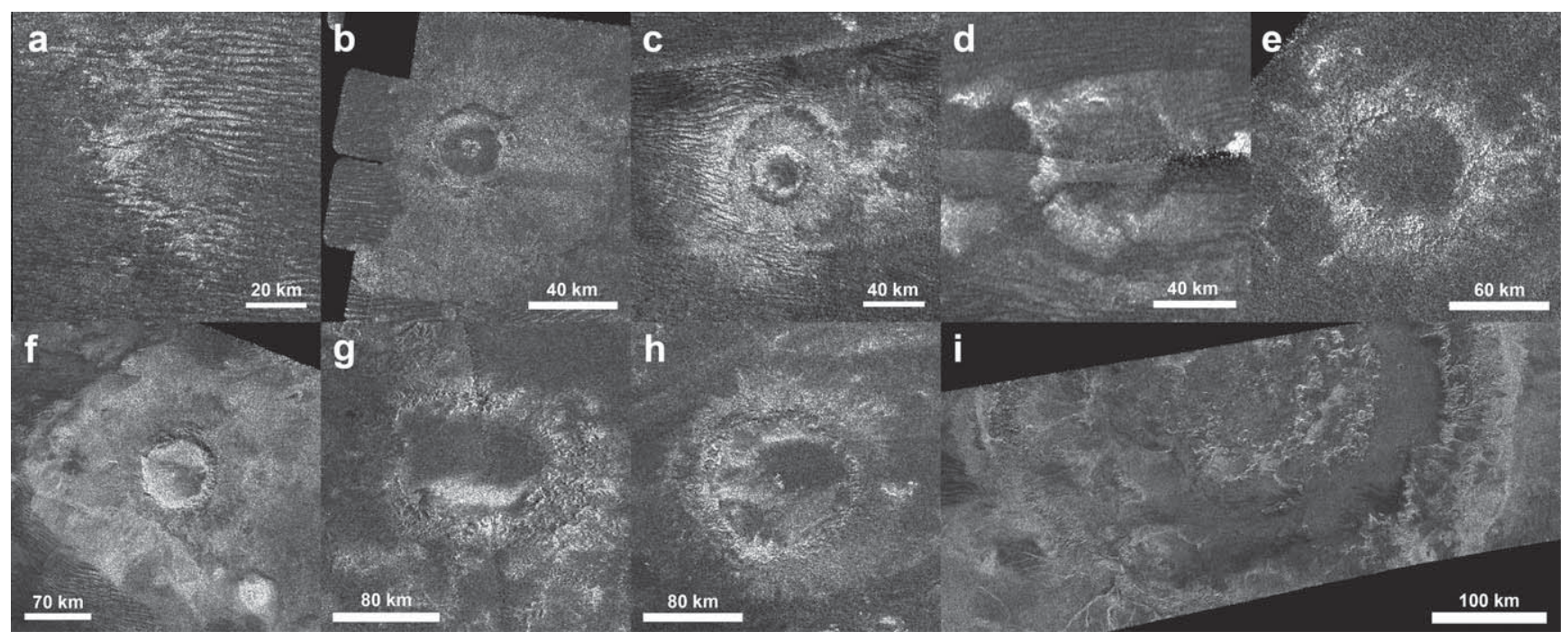

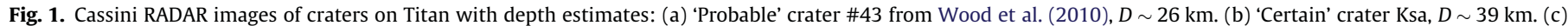
'Certain' crater Momoy, $D \sim 40 \mathrm{~km}$. This crater was discovered in Titan flyby T77, located near $11.6^{\circ} \mathrm{N}, 44.6^{\circ} \mathrm{W}$. Note the putative impact melt flows just to the east of the crater. (d) 'Probable' crater \#49 from Wood et al. (2010), D 60 km. (e) ‘Certain' crater Soi, D 78 km. (f) ‘Certain’ crater Sinlap, D 82 km. (g) ‘Nearly certain' crater Hano, $D \sim 100 \mathrm{~km}$. (h) ‘Certain’ crater Afekan, $D \sim 115 \mathrm{~km}$. (i) ‘Certain’ crater Menrva, $D \sim 425 \mathrm{~km}$. Each image is presented in simple cylindrical projection, and north is up.

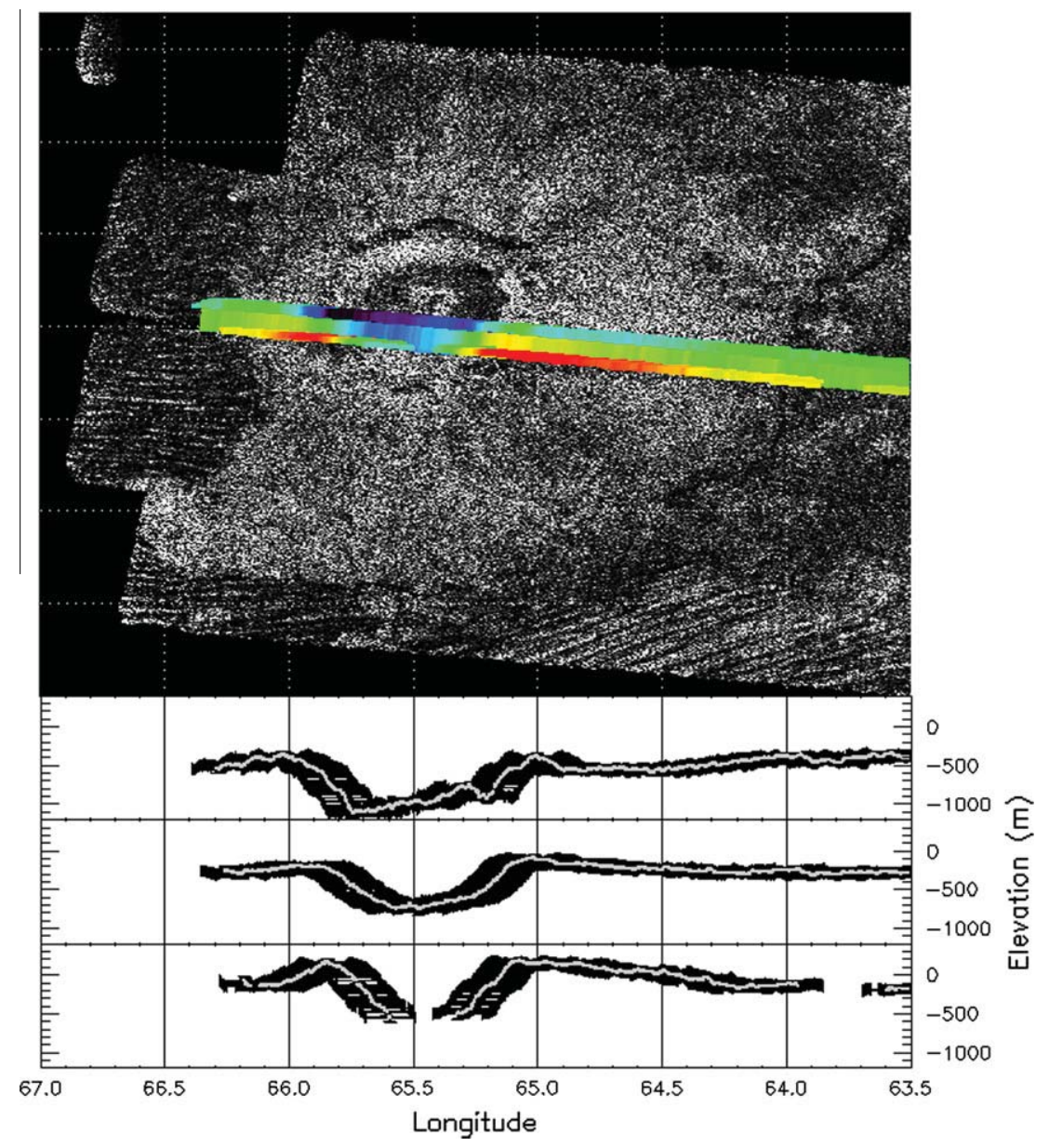

Fig. 2. (top) Ksa crater ( $D \sim 39 \mathrm{~km})$ as viewed by Cassini RADAR. North is up, and illumination is from the bottom. (bottom) Three topographic profiles through Ksa crater. The

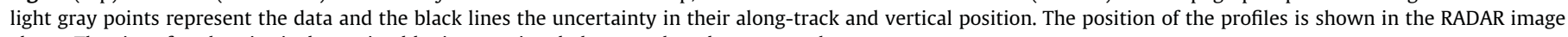
above. The size of each point is determined by its associated along-track and across-track errors. 


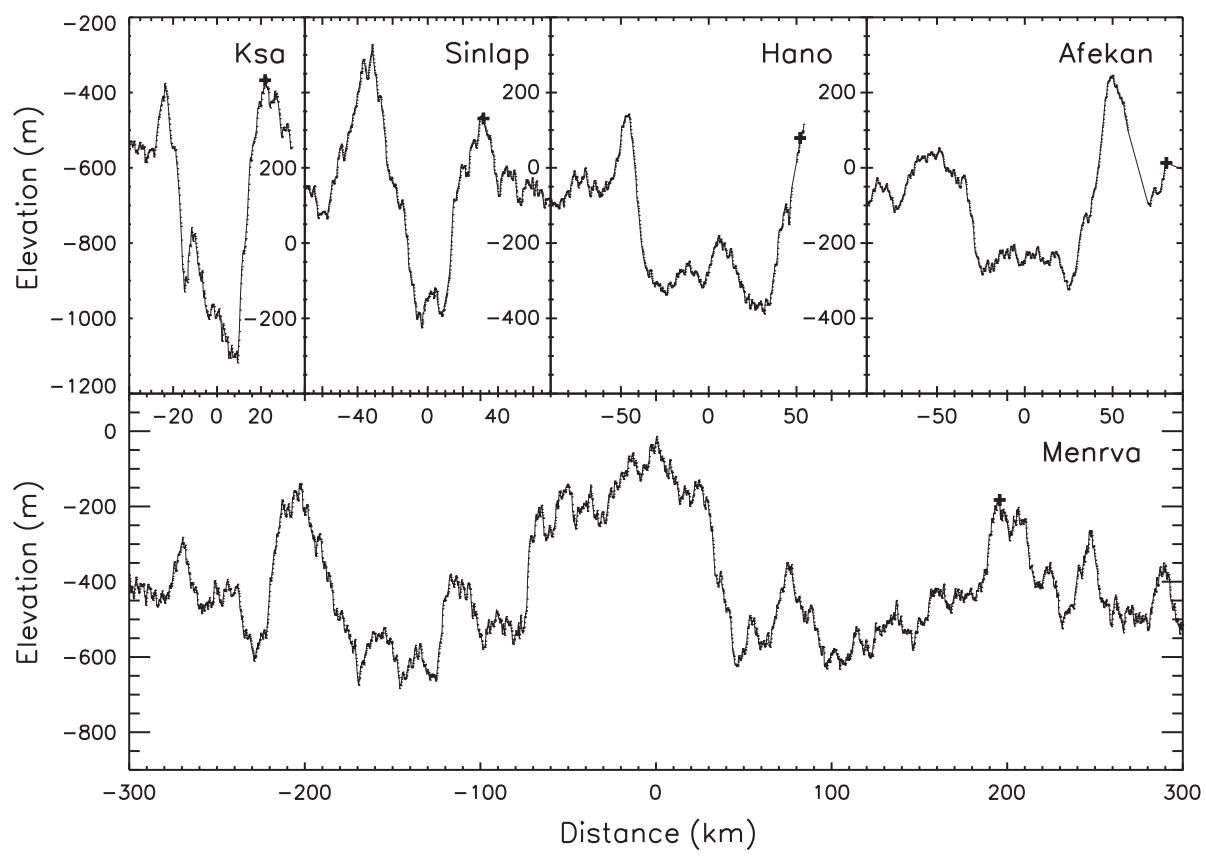

Fig. 3. Topographic profiles through five craters with corresponding SARTopo data and topography consistent with craters on other icy satellites: Ksa $(D \sim 39$ km), Sinlap $(D \sim 82 \mathrm{~km})$, Hano $(D \sim 100 \mathrm{~km})$, Afekan $(D \sim 115 \mathrm{~km})$ and Menrva $(D \sim 425 \mathrm{~km})$. The top four craters are plotted at the same spatial scale covered by Menrva for comparison. Representative along-track and vertical error bars are included in each plot.

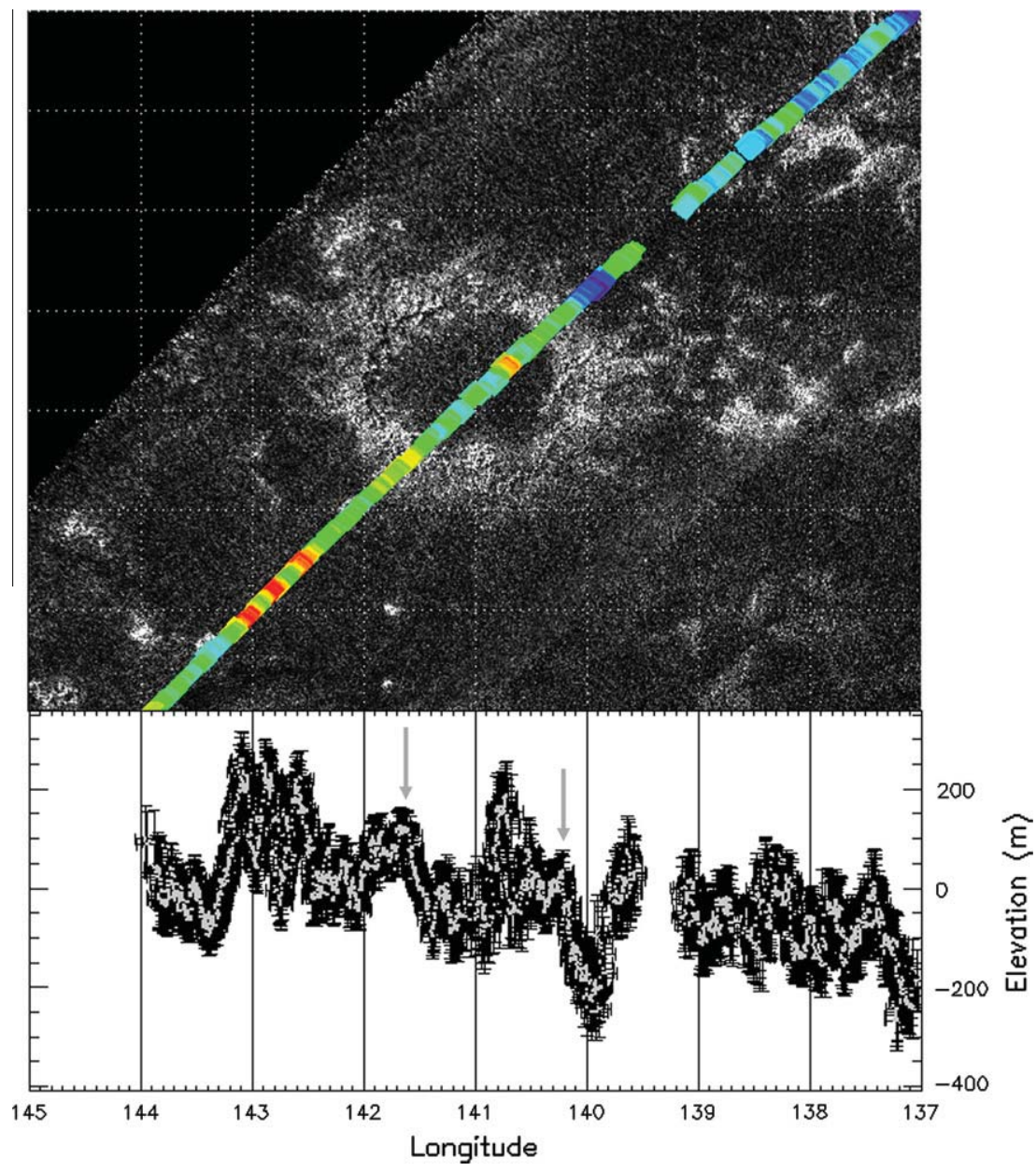

Fig. 4. (top) Soi ( $D \sim 78 \mathrm{~km}$ ), as viewed by Cassini RADAR. North is up, and illumination is from the bottom. (bottom) Topographic profile through Soi. The light gray points represent the data and the black lines the uncertainty in their along-track and vertical position. Gray arrows highlight the position of the western and eastern rims of the crater apparent in the Cassini RADAR image. 


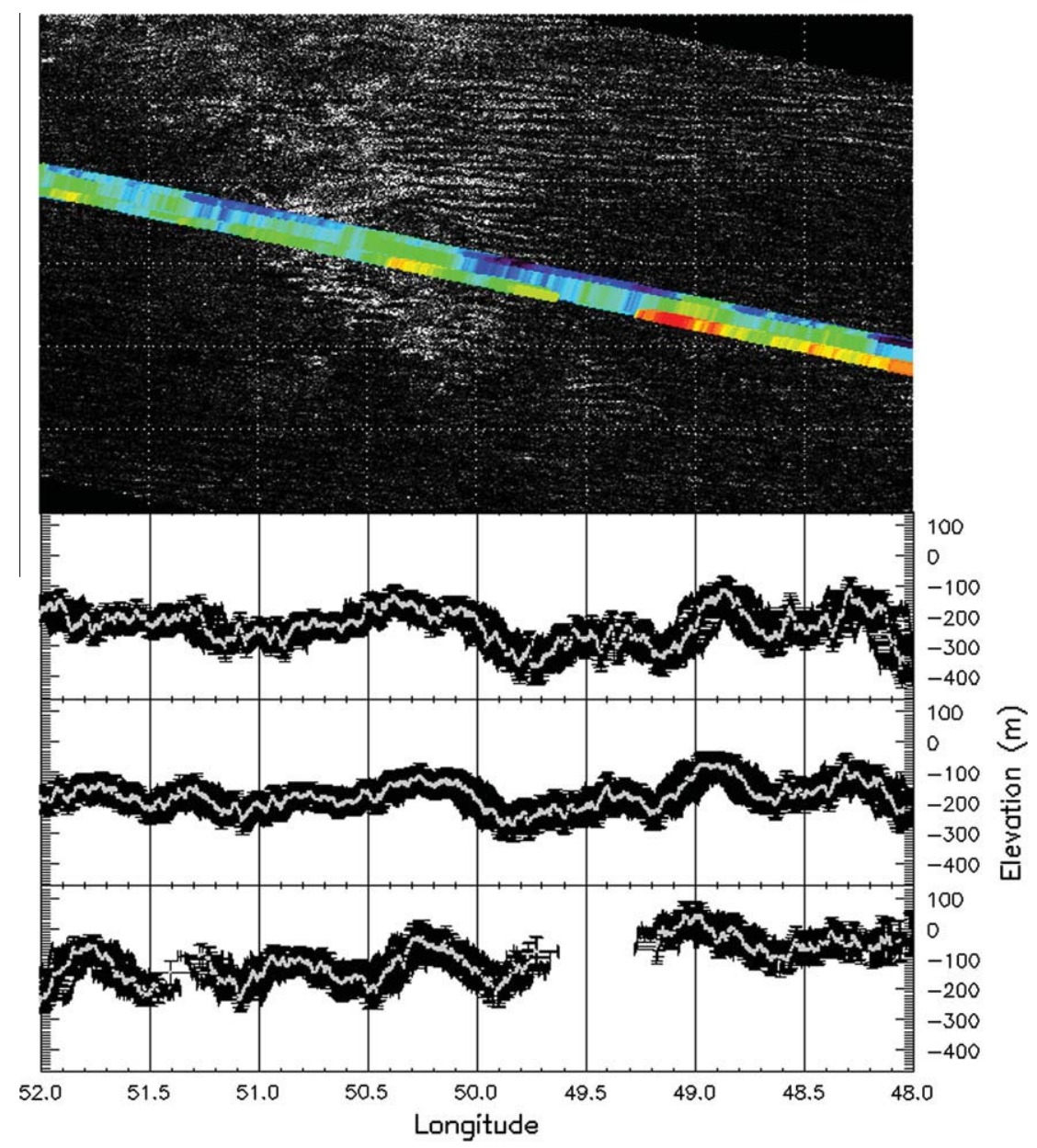

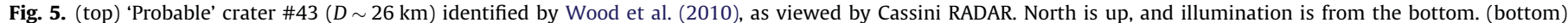

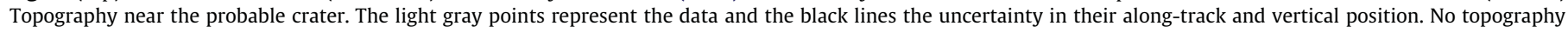
consistent with a crater is visible in this region, suggesting that the feature is not an impact crater or that it has been completely filled by sand.

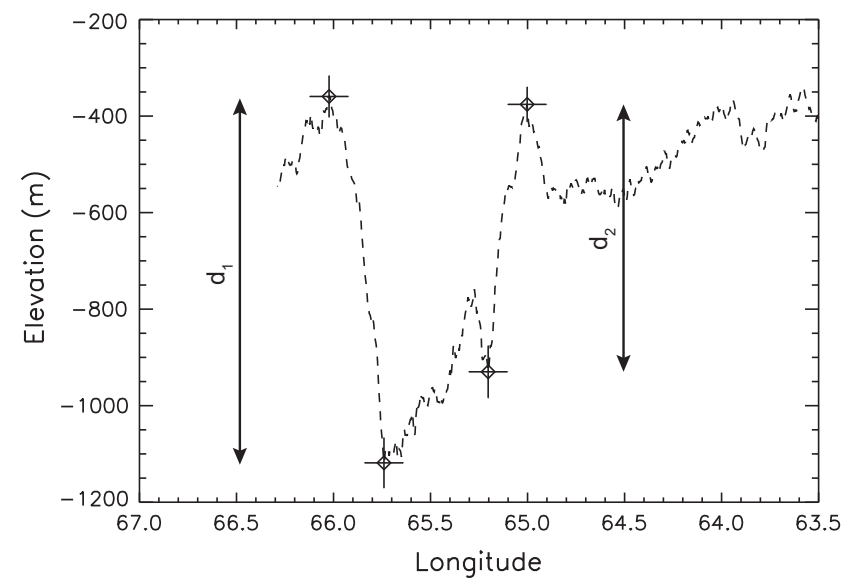

Fig. 6. Topographic profile of Ksa crater, marked with the location of the rim and floor elevations used to determine crater depth. The depths on either side of the crater, $d_{1}$ and $d_{2}$, were averaged to account for the natural variation of crater depth along the rim. Each point in this data set was averaged over a $\sim 10 \times 2 \mathrm{~km}$ wide area. This method tends to reduce crater depth, as adjacent topography is averaged with the rim maximum and floor minimum.

The two depths, $d_{1}$ and $d_{2}$ (where $d_{1}$ is taken to be the larger depth and $d_{2}$ the smaller) were then used to determine the average depth of the crater. We recognize that natural variations in depth around the rim of a crater, which are known to vary by 10-20\% on Mars (Stewart and Valiant, 2006), will likely dominate the uncertainty in the crater depth. Thus, to be as conservative as possible in our depth estimation, we report a range based on the largest depth consistent with our measurements, $d_{1}+\delta d_{1}$, and the smallest depth consistent with our measurements, $d_{2}-\delta d_{2}$. Stereo coverage is necessary to fully quantify the variations in depth around the rim of the crater, but thus far, Cassini RADAR has only acquired a stereo pair over Ksa crater. Preliminary analysis of this pair gives a depth of $750 \mathrm{~m}$, although the elevation of the rim varies by as much as $350 \mathrm{~m}$, representing a variation in depth of $\sim 45 \%$ (Kirk et al., 2012).

In the case of Soi, which was deemed a 'certain' crater by Wood et al. (2010), the SARtopo profile is nearly flat (Fig. 4; the position of the crater rim apparent in the SAR image is denoted by arrows). From morphological evidence in the SAR image, we judge that Soi is an impact crater (albeit, a highly modified one), and assign a depth based on the position of the inferred crater rim. We find that the western edge of Soi has a depth of $220 \pm 60 \mathrm{~m}$, whereas the eastern edge is essentially flat. There is also a topographic high near the middle of the crater, possibly a central peak similar to that observed at Ksa (Kirk et al., 2012). Averaging the depths on the two sides of the crater, we assign Soi a depth of $110 \pm 100 \mathrm{~m}$.

Depths were also estimated for two of Titan's craters by comparing the foreshortening of the near and far walls, in a technique we refer to as 'autostereo', which assumes a perfectly symmetric 
profile across the crater. The depth of one of these craters (Sinlap, diameter $D \sim 82 \mathrm{~km}$ ) agrees well with the corresponding SARTopo data, supporting the idea that Sinlap has a relatively flat floor. (An earlier estimate of $1300 \mathrm{~m}$ reported in Elachi et al. (2005) was lately found to be in error by a factor of two, resulting from confusion about the pixel scale of the image measured.) The second crater (Momoy, $D \sim 40 \mathrm{~km}$ ) was only recently discovered in RADAR imagery of the surface of Titan from Cassini flyby T77 (Fig. 1c). It is roughly the same depth $(680 \mathrm{~m}$ vs. $660 \mathrm{~m})$ as the similarly sized Ksa crater $(D \sim 39 \mathrm{~km})$.

\section{Comparisons to Ganymede}

Topography data were acquired for seven 'certain' or 'nearly certain' craters on Titan (Table 1). The depth-to-diameter ratios for these craters span the range $0.0012 \pm 0.0003$ (for the largest crater studied, Menrva, $D \sim 425 \mathrm{~km}$ ) to $0.017 \pm 0.004$ (for the smallest crater studied, Ksa, $D \sim 39 \mathrm{~km}$ ). We can compare these depths to fresh craters on Ganymede, which we assume represents a close 'airless' analogue to Titan (with few exogenic processes modifying the surface). We find that the depths of Titan craters are generally within the range of depths observed for similarlysized craters on Ganymede, but several hundreds of meters shal-
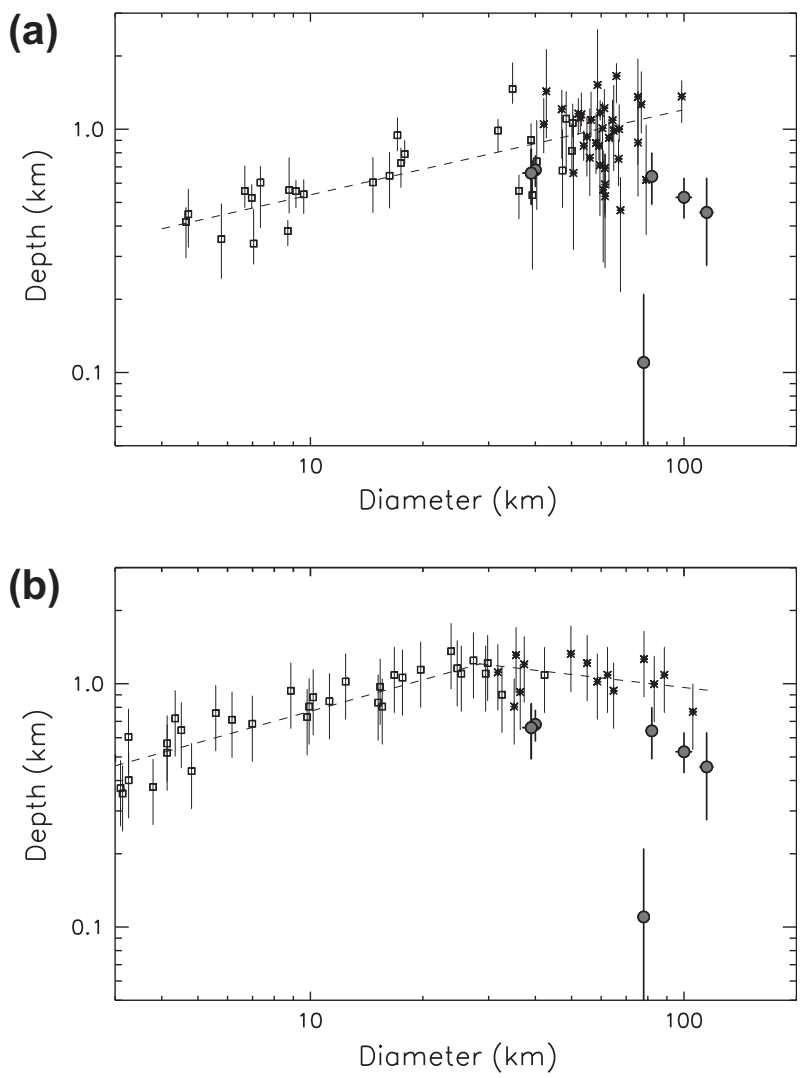

Fig. 7. Depth of craters on Titan (gray circles) derived from SARtopo measurements compared to similarly sized, fresh craters on Ganymede (central peaks, squares; central pits, stars) (a) from Bray et al. (2012) and (b) Schenk (2002). Dashed lines represent the power law fits to the Ganymede data reported in those two papers. Error bars in the Bray et al. (2012) data set incorporate both the range of natura variation in crater depth and a systematic error of $\sim 5 \%$. As shadow length measurements are only made from one quadrant of each crater, the natura variation in crater depth cannot be determined for the Schenk (2002) dataset. We do, however, include systematic errors of $30 \%$, which represent the maximum uncertainty in the shadow length measurements due to residual uncertainties in the control network and viewing geometry, as well as the limitation that the measurements can only be done on one side of the crater (Schenk, 1991). lower than the average depth vs. diameter trend for Ganymede (Fig. 7).

Given these data, we used the Anderson-Darling goodness-offit technique to evaluate the likelihood that the crater depths on Titan represent fresh Ganymede craters that have been altered by infilling. This technique evaluates the statistic $A^{2}$, which quantifies the difference between the observed data and model distribution functions, $F_{n}(x)$ and $F(x)$, respectively:

$A^{2}=n \int\left[\left(F_{n}(x)-F(x)\right)^{2} /(F(x)(1-F(x)))\right] d x$

The greater the difference between the data and model probability distribution, the larger $A^{2}$ becomes, increasing the probability of observing a given $A^{2}$ value by chance. In the case where the model $F(x)$ has no free parameters to adjust, the probability, $p=1-P\left(A^{2}<z\right)$, of observing a particular $A^{2}$ value can be obtained from standard tables (Lewis, 1961). Here, $P$ is the probability distribution function of the Anderson-Darling statistic described in Lewis (1961), and $z$ is the independent variable in that probability distribution function. A high $p$ value indicates that the observed result is likely if the null hypothesis being tested is true, whereas a low $p$ value indicates a significant contradiction to the null hypothesis.

Using this method, we evaluated two different hypotheses: (1) that the Titan craters we observe are fresh and have similar depths to fresh Ganymede craters of the same size and (2) that the observed depth of Titan's craters has been influenced by erosional infill after the crater was formed. We compared Titan depth measurements to Ganymede's fresh crater depths from two different data sets: the depths of craters on Ganymede reported in Bray et al. (2012), calculated from Galileo stereo DEMs (Fig. 7a), and the depths reported in Schenk (2002), calculated primarily from shadow length measurements (Fig. 7b). The depths in Bray et al. (2012) are notably shallower than those calculated by Schenk (2002), plotting parallel to, but below, the trend line in Schenk (2002). This difference can be explained by the averaging of the stereo data, which causes a decrease in measured crater depths. Similarly, the averaging of the Titan topography data over a $10 \mathrm{~km} \times w \mathrm{~km}$ region may reduce crater depths compared to the higher resolution Ganymede data (which have resolutions of several hundred meters to more than a kilometer per pixel). However, when we compare the depth determined from the Ksa DEM (Kirk et al., 2012) at resolutions similar to those on Ganymede $(1.4 \mathrm{~km} / \mathrm{pixel})$ to the depth obtained from SARTopo ( $\sim 10 \mathrm{~km} / \mathrm{pix}-$ el), we find little difference $(750 \pm 175 \mathrm{~m}$ vs. $660 \pm 170 \mathrm{~m})$. We thus judge that resolution alone cannot account for the several hundred meter difference in depth observed in the Ganymede and Titan data sets.

We now evaluate the two hypotheses listed above. Hypothesis 1 states that Titan's craters were selected from the depth distribution of fresh craters on Ganymede. For the Bray et al. (2012) data set, this hypothesis has a significance of $A^{2}=2.03$, or $p=0.09$, if one only considers the upper limits of the crater depths for those craters with 'recognizable' topographic profiles. This significance drops to zero if we include Soi, or consider only the lower limits of the crater depths, since no known fresh crater on Ganymede with $D>20 \mathrm{~km}$ has a depth less than $\sim 500 \mathrm{~m}$. For the Schenk (2002) data set, the significance is zero in either case. Hypothesis 2 states that the relative depths of Titan's craters are uniformly distributed between 0 (fresh) and 1 (completely infilled). Here, the relative depth, $R$, is given by $R(D)=1-d_{t}(D) / d_{g}(D)$, where $d_{t}(D)$ is the depth of a crater with diameter $D$ on Titan, and $d_{g}(D)$ is the depth of a crater with diameter $D$ on Ganymede, calculated from the empirical relation for $d$ determined by Schenk (2002) and the relation for $d / D$ determined by Bray et al. (2012). This hypothesis has a much higher significance than Hypothesis $1, A^{2}=0.931$, or $p=0.4$, for the Schenk (2002) data set, and $A^{2}=0.330$, or $p=0.92$, 
for the Bray et al. (2012) data set. If we only consider lower limits on depth, this hypothesis has a significance of $A^{2}=1.470$, or $p=0.2$, for the Schenk (2002) data set, and $A^{2}=0.949$, or $p=0.38$, for the Bray et al. (2012) data set. If we only consider upper limits on depth, this hypothesis has a significance of $A^{2}=1.341$, or $p=0.22$, for the Schenk (2002) data set, and $A^{2}=0.851$, or $p=0.45$, for the Bray et al. (2012) data set. These significances are increased further if we assume a uniform distribution between 0 and $x$, where $x<1$ as a consequence of there being a minimum detectable depth of $\sim 100 \mathrm{~m}$ in the SARtopo data set.

\section{Discussion}

Given the results of our analysis, we judge that it is very unlikely that Titan's craters have the same depth distribution as fresh craters on Ganymede. They are more consistent with a uniform distribution of relative depths between 0 (fresh) and 1 (completely infilled). Such a distribution suggests a modification process whose rate is constant with time, such as aeolian infilling (ForsbergTaylor et al., 2004). This interpretation is consistent with the location of the craters studied in this work, which are all (except Hano) within $30^{\circ}$ of Titan's equator, a region dominated by large sand seas (Lorenz and Radebaugh, 2009). In contrast, fluvial modification has an infilling rate that diminishes with time, as crater slopes diminish and the area of deposition on the crater floor increases. Craters in the Sinus Sabeus Quadrangle on Mars show a depth distribution consistent with fluvial modification, with a peak near $R=0.05$ (fresh craters) and another peak near $R=0.6-0.8$ (degraded craters) (Forsberg-Taylor et al., 2004). In this case, $R$ is defined as $R=(H-h) / H$, where $H$ represents the original fresh crater depth as estimated from relationships derived from MOLA topography (Bleacher et al., 2003) and $h$ is the actual crater depth. Only one crater with $R \sim 0.6-0.8$ was observed in our data set, suggesting either that fluvial modification is not the primary erosive process on Titan, or that the craters we are observing are very young and have not yet reached the state of degradation observed on Mars.

A uniform distribution of relative depths is also consistent with direct atmospheric sedimentation, but we judge this hypothesis to be less compelling given the expected photochemical production rates on Titan. The most abundant solid product of Titan's photochemistry is predicted to be acetylene, $\mathrm{C}_{2} \mathrm{H}_{2}$, and photochemical models predict a downward flux of $\mathrm{C}_{2} \mathrm{H}_{2}$ between $5.6 \times 10^{8}$ and $1.9 \times 10^{9}$ molecules $\mathrm{cm}^{-2} \mathrm{~s}^{-1}$ (Yung et al., 1984; Lara et al., 1994; Toublanc et al., 1995). This flux represents a sedimentation rate (assumed uniform over Titan) of $\sim 10-35 \mathrm{~m} / \mathrm{Gyr}$. Even if Titan's crater retention age is older than the $1 \mathrm{Ga}$ that current estimates suggest (Neish and Lorenz, 2012), it is unlikely that a layer of directly precipitated atmospheric sediment could account for the several hundred meters of infill we interpret to be present in Titan's craters.

We thus judge aeolian infill (which may represent horizontal surface transport of photochemical material that was originally deposited from the atmosphere) to be the more likely cause. This would represent an infilling rate of several hundred meters of sand per billion years, if sand transport rates have been constant on Titan over these timescales. If the dunes represent a younger surface feature, the inferred infilling rate could be much higher. On Earth, the floor of the crater Roter Kamm appears to have been filled by more than $300 \mathrm{~m}$ of sand on timescales less than a few million years (Fudali, 1973; Radebaugh et al., 2010). Thus, many more craters might be present but unidentified on Titan due to rapid aeolian infilling.

Another mechanism known to alter crater topography and reduce crater depths on icy satellites is viscous relaxation (Passey and Shoemaker, 1982; Dombard and McKinnon, 2006). Craters $>10 \mathrm{~km}$ in diameter on Ganymede show a range of relaxation states, from fresh craters to craters with more subdued topography and upbowed floors. However, given Titan's lower surface temperature ( $95 \mathrm{~K}$ vs. $120 \mathrm{~K}$ ), viscous relaxation is predicted to cause less than a $3 \%$ change in topography for craters with $D<125 \mathrm{~km}$ on Ti$\tan$ (Baugh and Brown, 2006), suggesting that viscous relaxation is not an important mechanism for modification of most craters on Titan. Nonetheless, craters near the polar regions of Ganymede, where surface temperatures approach $80 \mathrm{~K}$, do show evidence for relaxation (Dombard and McKinnon, 2006). In addition, recent measurements of the depths of large $(D>50 \mathrm{~km})$ craters on Rhea and Dione suggest that numerical models under-predict the amount of crater relaxation, indicating that these satellites might have experienced more heating early in their history than previously thought (Phillips et al., 2012).

Still, we judge it unlikely that viscous relaxation has been the primary form of crater modification on Titan. Viscous relaxation, like fluvial modification, has a modification rate that diminishes with time (see, for example, Fig. 9 in Dombard and McKinnon, 2006). This is because the strain rate associated with viscous flow is proportional to the depth of the crater (Melosh, 1989). As the crater relaxes and the depth decreases, the rate of modification will slow. We would therefore not expect a uniform distribution of relative depth values if viscous relaxation were the primary mechanism for crater modification on Titan. In addition, we would expect smaller craters to have systematically smaller relative depths, $R$, than larger craters, since larger craters relax faster than smaller craters (Passey and Shoemaker, 1982). This relationship is not observed for the Schenk (2002) data set, but further work will be required to determine whether it is consistent with the Bray et al. (2012) data set (Fig. 8). Of the craters with $D<125 \mathrm{~km}$ studied in this work, Afekan seems most consistent with a relaxed crater, showing evidence for an upbowed crater floor in the crater profile (Fig. 3).

Unlike its smaller counterparts, the largest crater observed on Titan, Menrva ( $D \sim 425 \mathrm{~km}$ ), would be expected to undergo significant topographic relaxation (assuming it did not breach the crust altogether, or that compositional differences such as the presence of methane clathrate do not make Titan's near-surface rheology substantially different from Ganymede's). On Ganymede, craters with $D>\sim 150 \mathrm{~km}$ are significantly shallower than smaller complex craters (Schenk et al., 2004). The largest preserved impact feature on Ganymede, Gilgamesh $(D \sim 590 \mathrm{~km})$, shares a similar topographic expression to that of Menrva. Gilgamesh has a central, domical surface rising up to $\sim 500 \mathrm{~m}$ and spanning $\sim 150 \mathrm{~km}$,

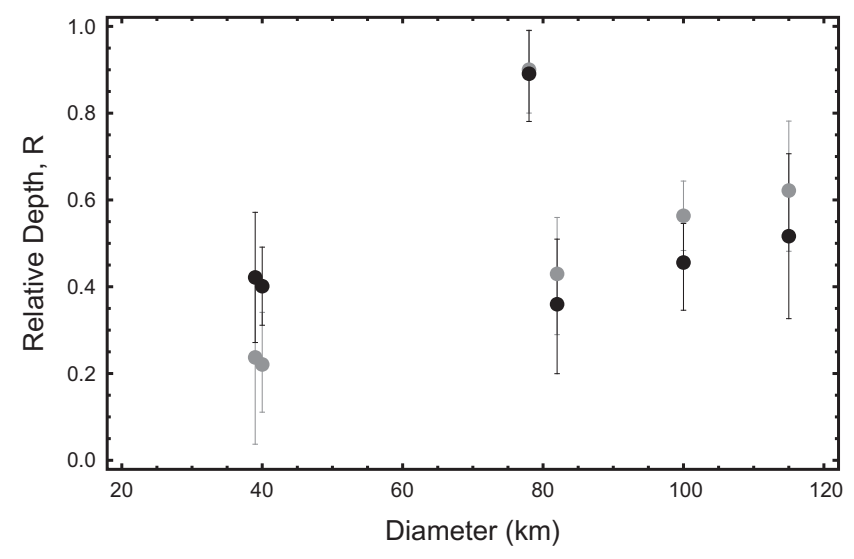

Fig. 8. Distribution of relative depth with crater diameter, calculated relative to the Ganymede depths from Bray et al. (2012) (gray) and Schenk (2002) (black). 
similar to that observed at Menrva (Fig. 3), though with an additional $\sim 1 \mathrm{~km}$ high scarp bounding the dome (Schenk et al., 1997). Gilgamesh's dome is then surrounded by a $225 \mathrm{~km}$ wide annular zone of hummocky terrain, bounded by an inward-facing scarp $\sim 1-\mathrm{km}$ high, which is presumed to be the basin rim. Beyond the apparent rim lies a second scarp $0.5-1 \mathrm{~km}$ high at a diameter of $750 \mathrm{~km}$, and a furrow at a diameter of $\sim 980 \mathrm{~km}$ which is concentric to Gilgamesh. This morphology gives Gilgamesh a resemblance to multi-ring basins on the Moon, such as Orientale (Passey and Shoemaker, 1982). Menrva's rim is somewhat more subdued than that of Gilgamesh, with a height of only several hundred meters, perhaps eroded by the fluvial processes evident in this region. It also lacks the exterior scarps observed at Gilgamesh, and thus cannot be classified as a multi-ring basin. It is possible that if such scarps existed in the plains beyond Menrva's rim, they would be buried by sediment and indistinguishable to Cassini RADAR. Numerical modeling of the formation and evolution of Menrva should be a productive direction for future research.

The Visual and Infrared Mapping Spectrometer (VIMS) provides compositional information for the crater floors on Titan, providing an independent means to distinguish between the different modification processes. VIMS has observed three craters on Titan in detail: the $82 \mathrm{~km}$ diameter Sinlap (Le Mouélic et al., 2008), the $90 \mathrm{~km}$ diameter Selk (Soderblom et al., 2010), and the $120 \mathrm{~km}$ diameter Paxsi (Buratti et al., 2012). Spectra of the floors of both Sinlap and Selk indicate they are composed of the 'dark blue' spectral unit, which has been interpreted as being enhanced in water-ice (Rodriguez et al., 2006; Barnes et al., 2007). Spectra of the floor of Paxsi indicate that it is composed of the 'dark brown' spectral unit, which is correlated with regions of dune fields (Soderblom et al., 2007). Thus, only one of the three craters studied is consistent with the presence of aeolian infill (although only one of these - Sinlap has the topographic data necessary for our depth analysis). The spectral properties suggest that Selk and Sinlap are fresh craters, or that they have been altered by viscous relaxation or fluvial modification, rather than aeolian infilling. Fluvial modification is most consistent with their morphologies (see, for example, Fig. 5 in Forsberg-Taylor et al. (2004)), and would act to deposit icy alluvium in the crater floor, consistent with their spectral properties. Taken together, Titan's craters seem more consistent with aeolian infill, but individual craters such as Sinlap may have experienced some fluvial modification.

In addition to the six certain craters discussed above, two 'probable' craters were found to have overlapping SARtopo data. Neither was observed to have topography consistent with a crater, but both features are located in Titan's sand seas, leading to the possibility that they have been completely infilled by aeolian deposits. Simple aeolian infilling tends to leave the crater rim largely clear of deposits, while the crater center becomes filled with a deposit roughly parabolic in cross-section (Forsberg-Taylor et al., 2004). Thus, we could observe a relatively pristine crater rim if these craters had been buried in sand. However, the topography data appear to be more consistent with a small hill around which sand has been diverted. Future stereo measurements of one or more of the probable craters would aid in determining whether they were caused by an impact.

Although we have assumed in this work that Titan's craters represent modified versions of Ganymede's fresh craters, there are a few possible differences between the crusts of Titan and Ganymede that might affect crater morphology. Two of these are (a) the presence of clathrates, such as methane clathrate (i.e., Tobie et al., 2006), or (b) the presence of liquid hydrocarbons in the crust (i.e., Kossacki and Lorenz, 1996). Models of Titan's interior evolution suggest that several of Titan's volatile species may be stored in the crust as clathrates (Tobie et al., 2012). Indeed, the outgassing of methane clathrates in Titan's upper crust has been suggested as a possible mechanism for replenishing Titan's photochemically unstable atmosphere (Tobie et al., 2006). Experiments have shown that methane clathrate hydrate is very rigid compared to water ice. At temperatures just below the freezing point of water, methane hydrate is over 20 times stronger than water ice at a given strain rate, and the contrast increases at lower temperatures (Durham et al., 2003). In general, for projectiles with the same characteristics, stronger targets will yield smaller craters than weaker targets (e.g., Wünnemann et al., 2011). So if Titan's crust consists of a thick layer of methane clathrate, we may expect craters on Titan to be smaller than those formed on Ganymede by comparable projectiles. However, models indicate that the clathrate layer, if present, should be relatively thin $(<10 \mathrm{~km})$ compared to the thick, water ice crust below ( $>50 \mathrm{~km}$ ) (Tobie et al., 2006), although the details of enclathration and retention of porosity (which will also influence strength) have not been explored. This scenario - impacts into a strong layer of methane clathrate over a weaker layer of water ice - has not been studied with impact hydrocodes. However, simulations into layered basalt show that the inclusion of thin layers of stronger basalt (two orders of magnitude stronger than normal basalt) does not have any significant effects on crater dimensions (Senft and Stewart, 2007). If clathrates are not present on Titan, or are only present as thin layers, this should have a relatively minor effect on crater dimensions compared to those on Ganymede. Thick layers of clathrates, however, may have an observable effect on crater diameter, and we thus judge this to be an area worthy of further study.

The presence of liquids on the surface and in the near subsurface can also cause extensive modification to crater shape, as is observed on Earth (e.g., Collins and Wünnemann, 2005; Ormö et al., 2006). In the case of Titan, liquids consist of hydrocarbons, either as wet unconsolidated sediments (such as those observed at the Huygens landing site; Niemann et al., 2005) or shallow marine environments (such as the lakes observed at the north and south poles; Stofan et al., 2007). Craters formed in similar environments on the Earth lack any significant surface topography, including the absence of a raised rim, as poorly consolidated, water-saturated sediments slump into the crater, generating a much larger structure than is expected from the amount of kinetic energy released from the impact (Collins and Wünnemann, 2005). In addition, sedimentation subsequent to the impact has covered or subdued the topographic expressions of all known marine-target craters on Earth (although buried craters can later be exposed at the surface by erosion, i.e., Lockne crater in Sweden, Lindström et al., 1996). It is possible that the lack of topography associated with marine-target impacts may help to explain the relative dearth of impact craters observed near the poles of Titan (Wood et al., 2010). If Titan's polar regions are saturated by liquid hydrocarbons, craters formed in those regions may lack any recognizable topographic expression.

\section{Summary}

In summary, our new observations of craters on Titan suggest that:

(1) The depths of Titan's craters are within the range of depths observed for similarly-sized fresh craters on Ganymede, but several hundreds of meters shallower than Ganymede's average depth vs. diameter trend.

(2) There is less than a $10 \%$ probability that Titan's craters have a depth distribution that is consistent with the depth distribution of fresh craters on Ganymede.

(3) The differences in depth appear most consistent with aeolian infilling, although some amount of viscous relaxation and fluvial modification may have contributed to the modification of Titan's craters. 


\section{Acknowledgments}

We wish to acknowledge the Cassini RADAR Team for acquiring and processing the data presented here. C.N., R.K., R.L., B.S., and E.T. further thank the Cassini Project for financial support. A.H. was funded by the Miller Institute for Basic Research in Science. This work was also partially supported by an appointment to the NASA Postdoctoral Program at the Goddard Space Flight Center, administered by Oak Ridge Associated Universities through a contract with NASA to C.N., and a portion of this research was carried out at the Jet Propulsion Laboratory, California Institute of Technology, under a Contract with the National Aeronautics and Space Administration. We thank O. Abramov and two anonymous reviewers for comments that helped to improve the manuscript.

\section{References}

Barnes, J.W., Brown, R.H., Soderblom, L., Buratti, B.J., Sotin, C., Rodriguez, S., Le Mouélic, S., Baines, K.H., Clark, R., Nicholson, P., 2007. Global-scale surface spectral variations on Titan seen from Cassini/VIMS. Icarus 186, 242-258.

Baugh, N., Brown, R.H., 2006. Modeling viscoelastic crater relaxation in ice with applications to Titan. Bull. Am. Astron. Soc. 38, 587.

Bleacher, J.E., Sakiomoto, S.E.H., Garvin, J.B., Wong, M., 2003. Deflation/erosion rates for the Parva Member, Dorsa Argentea Formation and implications for the south polar region of Mars. J. Geophys. Res. 108 (E7), 5075.

Bray, V.J., Collins, G.S., Morgan, J.V., Schenk, P.M., 2008. The effect of target properties on crater morphology: Comparison of central peak craters on the Moon and Ganymede. Meteorit. Planet. Sci. 43, 1979-1992.

Bray, V.J., Schenk, P.M., Melosh, H.J., Morgan, J.V., Collins, G.S., 2012. Ganymede crater dimensions - Implications for central peak and central pit formation and development. Icarus 217, 115-129.

Buratti, B.J. et al., 2012. A newly discovered impact crater in Titan's Senkyo: Cassini VIMS observations and comparison with other impact features. Planet. Space Sci. 60, 18-25.

Collins, G.S., Wünnemann, K., 2005. How big was the Chesapeake Bay impact? Insight from numerical modeling. Geology 33, 925-928.

Dombard, A.J., McKinnon, W.B., 2006. Elastoviscoplastic relaxation of impact crater topography with application to Ganymede and Callisto. J. Geophys. Res. 111 E01001.

Durham, W.B., Kirby, S.H., Stern, L.A., Zhang, W., 2003. The strength and rheology of methane clathrate hydrate. J. Geophys. Res. 108, 2182.

Elachi, C. et al., 2005. Cassini radar views the surface of Titan. Science 308, 970-974.

Forsberg-Taylor, N.K., Howard, A.D., Craddock, R.A., 2004. Crater degradation in the martian highlands: Morphometric analysis of the Sinus Sabaeus region and simulation modeling suggest fluvial processes. J. Geophys. Res. 109, E05002.

Fudali, R.F., 1973. Rotor Kamm: Evidence for an impact origin. Meteoritics 8, 245257.

Hayes, A.G., Aharonson, O., Lunine, J.I., Kirk, R.L., Zebker, H.A., Wye, L.C., Lorenz, R.D., Turtle, E.P., Paillou, P., Mitri, G., Wall, S.D., Stofan, E.R., Mitchell, K.L., Elachi, C., Cassini RADAR Team, 2011. Transient surface liquid in Titan's polar regions from Cassini. Icarus 211, 655-671.

Holsapple, K.A., Housen, K.R., 2007. A crater and its ejecta: An interpretation of Deep Impact. Icarus 187, 345-356.

Iess, L. et al., 2010. Gravity field, shape, and moment of inertia of Titan. Science 327, 1367-1369.

Kirk, R.L. et al., 2012. Topographic mapping of Titan: Latest results. Lunar Planet. Sci. 43. Abstract 2759.

Korycansky, D.G., Zahnle, K.J., 2005. Modeling crater populations on Venus and Titan. Planet. Space Sci. 53, 695-710.

Kossacki, K.J., Lorenz, R.D., 1996. Hiding Titan's ocean: Densification and hydrocarbon storage in an icy regolith. Planet. Space Sci. 44, 1029-1037.

Lara, L.M., Lorenz, R.D., Rodrigo, R., 1994. Liquids and solids on the surface of Titan: Results of a new photochemical model. Planet. Space Sci. 42, 5-14.

Le Mouélic, S. et al., 2008. Mapping and interpretation of Sinlap crater on Titan using Cassini VIMS and RADAR data. J. Geophys. Res. 113, E04003.

Lewis, P.A.W., 1961. Distribution of the Anderson-Darling statistic. Ann. Math. Stat. 32, 1118-1124

Lindström, M., Sturkell, E.F.F., Törnberg, R., Ormö, J., 1996. The marine impact crater at Lockne, central Sweden. Geol. Foren. Stock. For. 118, 193-206.

Lorenz, R.D., Radebaugh, J., 2009. Global pattern of Titan's dunes: Radar survey from the Cassini prime mission. Geophys. Res. Lett. 36, L03202.

Lorenz, R.D. et al., 2006. The sand seas of Titan: Cassini RADAR observations of longitudinal dunes. Science 312, 724-727.
Lorenz, R.D. et al., 2008. Fluvial channels on Titan: Initial Cassini RADAR observations. Planet. Space Sci. 56, 1132-1144.

Melosh, H.J., 1989. Impact Cratering: A Geologic Process. Oxford University Press, Oxford, UK.

Neish, C.D., Lorenz, R.D., 2012. Titan's global crater population: A new assessment Planet. Space Sci. 60, 26-33.

Niemann, H.B. et al., 2005. The abundances of constituents of Titan's atmosphere from the GCMS instrument on the Huygens probe. Nature 438, 779-784.

Nimmo, F., Bills, B.G., 2010. Shell thickness variations and the long-wavelength topography of Titan. Icarus 208, 896-904.

Ormö, J., Lindstrom, M., Lepinette, A., Martinez-Frias, J., Diaz-Martinez, E., 2006. Cratering and modification of wet-target craters: Projectile impact experiments and field observations of the Lockne marine-target crater (Sweden). Meteorit. Planet. Sci. 41, 1605-1612.

Passey, Q.R., Shoemaker, E.M., 1982. Craters and basins on Ganymede and Callisto Morphological indicators of crustal evolution. In: Morrison, D., Matthews, M.S. (Eds.), Satellites of Jupiter. University of Arizona Press, Tucson, AZ, pp. 379-434.

Phillips, C.B., Hammond, N.P., Robuchon, G., Nimmo, F., Beyer, R., Roberts, J., 2012. Stereo imaging, crater relaxation, and thermal histories of Rhea and Dione. Lunar Planet. Sci. 43. Abstract 2571.

Radebaugh, J., Lorenz, R., Farr, T., Paillou, P., Savage, C., Spencer, C., 2010. Linear dunes on Titan and Earth: Initial remote sensing comparisons. Geomorphology $121,122-132$.

Rodriguez, S. et al., 2006. Cassini/VIMS hyperspectral observations of the Huygens landing site on Titan. Planet. Space Sci. 54, 123-137.

Schenk, P.M., 1991. Ganymede and Callisto: Complex crater formation and planetary crusts. J. Geophys. Res. 96, 15635-15664.

Schenk, P.M., 2002. Thickness constraints on the icy shells of the Galilean satellites from a comparison of crater shapes. Nature 417, 419-421.

Schenk, P., McKinnon, W., Moore, J., 1997. Stereo topography of Valhalla and Gilgamesh. Lunar Planet. Sci. 28. Abstract 1775.

Schenk, P.M., Chapman, C., Moore, J., Zahnle, K., 2004. Ages and interiors: The cratering record of the Galilean satellites. In: Bagenal, F., McKinnon, W.B. (Eds.), Jupiter: The Planet, Satellites, and Magnetosphere. Cambridge University Press, Cambridge, UK, pp. 427-456.

Senft, L.E., Stewart, S.T., 2007. Modeling impact cratering in layered surfaces. J. Geophys. Res. 112, E11002.

Soderblom, L. et al., 2007. Correlations between Cassini VIMS spectra and RADAR SAR images: Implications for Titan's surface composition and the character of the Huygens Probe landing site. Planet. Space Sci. 55, 2025-2036.

Soderblom, J.M., Brown, R.H., Soderblom, L.A., Barnes, J.W., Jaumann, R., Le Mouélic, S., Sotin, C., Stephan, K., Baines, K.H., Buratti, B.J., Clark, R.N., Nicholson, P.D., 2010. Geology of the Selk crater region on Titan from Cassini VIMS observations. Icarus 208, 905-912.

Spohn, T., Schubert, G., 2003. Oceans in the icy Galilean satellites of Jupiter? Icarus $161,456-467$.

Stewart, S.T., Valiant, G.J., 2006. Martian subsurface properties and crater formation processes inferred from fresh impact crater geometries. Meteorit. Planet. Sci. 41, 1509-1537.

Stiles, B.W., Hensley, S., Gim, Y., Bates, D.M., Kirk, R.L., Hayes, A., Radebaugh, J., Lorenz, R.D., Mitchell, K.L., Callahan, P.S., Zebker, H., Johnson, W.T.K., Wall, S.D., Lunine, J.I., Wood, C.A., Janssen, M., Pelletier, F., West, R.D., Veeramacheneni, C., Cassini RADAR Team, 2009. Determining Titan surface topography from Cassini SAR data. Icarus 202, 584-598.

Stofan, E.R. et al., 2007. The lakes of Titan. Nature 445, 61-64.

Tobie, G., Lunine, J.I., Sotin, C., 2006. Episodic outgassing as the origin of atmospheric methane on Titan. Nature 440, 61-64.

Tobie, G., Gautier, D., Hersant, F., 2012. Titan's bulk composition constrained by Cassini-Huygens: Implication for internal outgassing. Astrophys. J. 752, 125.

Tomasko, M.G. et al., 2005. Rain, winds and haze during the Huygens probe's descent to Titan's surface. Nature 438, 765-778.

Toublanc, D., Parisot, J.P., Brillet, J., Gautier, D., Raulin, F., McKay, C.P., 1995. Photochemical modeling of Titan's atmosphere. Icarus 113, 2-26.

Wood, C.A., Lorenz, R., Kirk, R., Lopes, R., Mitchell, K., Stofan, E., Cassini RADAR Team, 2010. Impact craters on Titan. Icarus 206, 334-344.

Wünnemann, K., Nowka, D., Collins, G.S., Elbeshausen, D., Bierhaus, M., 2011. Scaling of impact crater formation on planetary surfaces: Insights from numerical modeling. In: Hiemaier, S. (Ed.), Proceedings of the 11th Hypervelocity Impact Symposium, Fraunhofer, Freiburg, Germany, pp. 1-16.

Yung, Y.L., Allen, M., Pinto, J.P., 1984. Photochemistry of the atmosphere of Titan: Comparison between model and observations. Astrophys. J. Suppl. Ser. 55, 465506.

Zahnle, K., Schenk, P., Levison, H., Dones, L., 2003. Cratering rates in the outer Solar System. Icarus 163, 263-289.

Zebker, H.A., Gim, Y., Callahan, P., Hensley, S., Lorenz, R., Cassini RADAR Team, 2009 Analysis and interpretation of Cassini Titan radar altimeter echoes. Icarus 200, 240-255. 